\title{
Statin Use, Diabetes Incidence and Overall Mortality in Normoglycemic and Impaired Fasting Glucose Patients
}

\author{
M. Regina Castro, $M D^{7}$, Gyorgy Simon, PhD', Stephen S. Cha, $M S^{3}$, Barbara P. Yawn, MD', \\ L. Joseph Melton III, $M D^{5}$, and Pedro J. Caraballo, $M D^{6,7}$
}

'Department of Medicine, Division of Endocrinology, Mayo Clinic, Rochester, MN, USA; ${ }^{2}$ Institute for Health Informatics, University of Minnesota, Minneapolis, MN, USA; ${ }^{3}$ Department of Health Sciences Research, Division of Biomedical Statistics \& Informatics, Mayo Clinic, Rochester, MN, USA; ${ }^{4}$ Department of Research, Olmsted Medical Center, Rochester, MN, USA; ${ }^{5}$ Department of Health Sciences Research, Division of Epidemiology, Mayo Clinic, Rochester, MN, USA; ${ }^{6}$ Department of Medicine, Division of General Internal Medicine, Mayo Clinic, Rochester, MN, USA; ${ }^{7}$ Mayo Clinic, Rochester, MN, USA.

BACKGROUND: The association between the use of statins and the risk of diabetes and increased mortality within the same population has been a source of controversy, and may underestimate the value of statins for patients at risk. OBJECTIVE: We aimed to assess whether statin use increases the risk of developing diabetes or affects overall mortality among normoglycemic patients and patients with impaired fasting glucose (IFG).

DESIGN AND PARTICIPANTS: Observational cohort study of 13,508 normoglycemic patients $(n=4460 ; 33 \%$ taking statins) and 4563 IFG patients ( $n=1865 ; 41 \%$ taking statin) among residents of Olmsted County, Minnesota, with clinical data in the Mayo Clinic electronic medical record and at least one outpatient fasting glucose test between 1999 and 2004. Demographics, vital signs, tobacco use, laboratory results, medications and comorbidities were obtained by electronic search for the period 1999-2004. Results were analyzed by Cox proportional hazards models, and the risk of incident diabetes and mortality were analyzed by survival curves using the Kaplan-Meier method.

MAIN MEASURES: The main endpoints were new clinical diagnosis of diabetes mellitus and total mortality.

KEY RESULTS: After a mean of 6 years of follow-up, statin use was found to be associated with an increased risk of incident diabetes in the normoglycemic (HR 1.19; $95 \%$ CI, 1.05 to $1.35 ; p=0.007$ ) and IFG groups (HR 1.24; $95 \% \mathrm{CI}, 1.11$ to $1.38 ; p=0.0001)$. At the same time, overall mortality decreased in both normoglycemic (HR 0.70; $95 \%$ CI, 0.66 to $0.80 ; p<0.0001$ ) and IFG patients (HR $0.77,95 \%$ CI, 0.64 to $0.91 ; p=0.0029$ ) with statin use.

CONCLUSION: In general, recommendations for statin use should not be affected by concerns over an increased risk of developing diabetes, since the benefit of reduced mortality clearly outweighs this small (19-24\%) risk.

KEY WORDS: statins; diabetes; mortality; prediabetes; impaired fasting glucose.

J Gen Intern Med 31(5):502-8

DOI: $10.1007 /$ s11606-015-3583-0

(C) Society of General Internal Medicine 2016

Received April 30, 2015

Revised September 18, 2015

Accepted December 16, 2015

Published online February 5, 2016

\section{INTRODUCTION}

Recent publications have shown an association between the use of statins and the risk of developing diabetes mellitus, although the clinical relevance of this association has been debated. ${ }^{1-3}$ However, the benefits of statin therapy in reducing cardiovascular risk in both diabetic and non-diabetic patients are unquestionable, and have been clearly demonstrated in multiple studies, with more than 500,000 patient-years of treatment. Such benefits are greatest in individuals with the highest cardiovascular risk. ${ }^{4,5}$ The West of Scotland Coronary Prevention Study (WOSCOPS) was the first randomized clinical trial (RCT) to indicate a possible link between statins use and the risk of diabetes, suggesting that pravastatin therapy was associated with a $30 \%$ reduction in the risk of developing diabetes. ${ }^{1}$ Several subsequent RCTs have failed to confirm this protective effect. $^{6-9}$ Rather, a possible adverse effect of statins on the incidence of diabetes was initially reported as a post hoc incidental finding in an RCT by Ridker et al. in $2008 .{ }^{10}$ This finding prompted further review, and multiple studies have since been published, but results have been conflicting, with one meta-analysis including six RCTs showing no clear association, ${ }^{11}$ while two larger and more recent reviews pointed to an increased risk of diabetes. ${ }^{3,12}$ One of these was a metaanalysis that included a total of 13 RCTs, each with more than 1000 patients, in which various statins were used for periods greater than 1 year, ${ }^{3}$ while the other was a review of three large RCTs investigating high-dose atorvastatin $(80 \mathrm{mg}) .{ }^{12}$ Furthermore, it is unclear whether statin use further accelerates progression to overt diabetes in patients already at increased risk, e.g., patients with impaired fasting glucose (IFG) or impaired glucose tolerance (IGT), although the use of statins appears to be associated with increased risk of new-onset diabetes in some patients (IGT with other associated cardiovascular risk factors). ${ }^{13}$ Of concern, however, is whether the welldocumented beneficial effect of statins in reducing cardiovascular risk ${ }^{6}$ is negated by an incremental increase in the risk of developing diabetes, particularly for primary prevention in patients at lower risk where the benefit is less clear. ${ }^{14-18}$ One must also question whether the risk of diabetes and that of cardiovascular events and death carry the same weight. ${ }^{19}$ In 
this study, we aimed to assess whether the use of statins increases the risk of developing diabetes and whether it affects total mortality in patients with IFG and in normoglycemic patients.

\section{RESEARCH DESIGN AND METHODS}

\section{Study Setting and Subjects}

The Mayo Clinic, located in Rochester, MN, provides primary care to a large proportion of the residents of Olmsted County, MN. The facility has a comprehensive electronic medical record (EMR) system that includes laboratory results, clinical diagnoses and clinical notes, which in the outpatient setting are organized in sections (e.g., chief complaint, history of present illness, allergies, medications), thus facilitating electronic search of their content. This information is also part of the Rochester Epidemiology Project (REP), a unique research infrastructure that links the Mayo medical records with those of other providers who serve the local population, most notably the Olmsted Medical Center, and allows follow-up of the population over time.

After approval by the Mayo Clinic Institutional Review Board, we used these databases to identify all eligible adult ( $\geq 18$ years) Olmsted County residents who visited the Mayo Clinic between January 1, 1999, and December 31, 2004, and who had clinical follow-up after January 1, $2005(n=85,132)$. Residents who had not provided authorization for use of their medical records in research were not included. We then excluded patients with a clinical diagnosis of diabetes (ICD codes searched using REP resources) or who met biochemical criteria for diabetes (fasting glucose $\geq 126 \mathrm{mg} / \mathrm{dL}$ ). We also excluded patients who were not taking any medication, as these patients are generally younger and healthier, and a substantial portion of them had not had laboratory tests performed, and thus fasting glucose values were missing. By excluding these patients, we assumed our sample consisted of comparable subjects. A total of 18,071 adult non-diabetic Olmsted County residents who were taking some medication and had at least one outpatient fasting glucose test between 1999 and 2004 constituted our study sample.

\section{Data Collection and Follow-up}

We collected baseline clinical characteristics using electronic searches: date of birth and gender were obtained from the REP database, as were clinical diagnoses (ICD codes) of obesity, hypertension, hyperlipidemia, renal failure, congestive heart failure, ischemic heart disease, cerebrovascular disease and peripheral vascular disease. For vital signs (weight, height, body mass index (BMI), blood pressure, pulse) and laboratory values (total cholesterol, triglycerides, HDL cholesterol, LDL cholesterol and creatinine), we used the mean $( \pm \mathrm{SD})$ of all available results for each subject in the Mayo EMR between January 1, 1999, and December 31, 2004. Tobacco use and medications were assessed using natural language processing, searching the social history and medication section of the EMR between 1999 and 2004. Exposure to statins was defined as the presence of any generic or brand-name statin, alone or in combination with other medications, in the medication list during the study period. We used the highest outpatient fasting plasma glucose level recorded between January 1, 1999, and December 31, 2004, to define normoglycemia $(<100 \mathrm{mg} / \mathrm{dL})$ and IFG (100 to $125 \mathrm{mg} / \mathrm{dL}$ ) according to current American Diabetes Association criteria. We used plasma glucose measures defined as "fasting" and collected between 6:00 a.m. and noon in the outpatient setting. Blood glucose defined as "random glucose" or any measurements collected in the emergency department or any hospital location was excluded. Patients were then followed in time through the REP resources until death or the last visit to Mayo Clinic to identify new onset of clinical diagnosis of diabetes mellitus.

\section{Statistical Analysis}

We divided the patients with a history of medication use into two groups according to their baseline risk of diabetes (normoglycemic vs. IFG), and we then compared those patients exposed to statins vs. those without statin exposure within each group. Patient characteristics were reported as mean \pm SD for continuous variables and frequency (percentage) for categorical variables, and were compared by two-sample $t$ tests and Pearson chi-square tests between statin users and non-statin users when appropriate. BMI, total cholesterol, HDL, LDL and triglycerides were missing for $7 \%$ of the population. However, we did not impute data, as these data were not missing at random, and they were predominantly for younger and healthier patients who were not taking medications.

Cox proportional hazards models were constructed to model total mortality and diabetes as two separate endpoints. Predictors included known risk factors for mortality and diabetes (Table 1). Since lipid health is an important factor in these endpoints, and our intervention of interest (statin treatment) concerns lipid health in particular, correctly taking lipid health into account is of paramount importance. The effects of statins and hyperlipidemia are highly conflated. Nearly half of the patients with a diagnosis of hyperlipidemia are taking lipid-lowering drugs, and $92 \%$ of these drugs are statins. From a modeling perspective, the effect of statins and that of hyperlipidemia are difficult to separate, but we can reliably estimate their combined effect. To this end, we constructed a propensity score model, which we termed "baseline lipid risk," to assess the patients' baseline (January 1, 2005) lipid health. Specifically, we defined it as the log odds of the necessity to use any cholesterol drug or the presence of a hyperlipidemia diagnosis code. The independent variables included lipid panel (LDL, HDL, triglycerides), blood pressure, BMI, the use of hypertension drugs and demographics (age and gender). Backwards elimination was applied to select a set of statistically significant predictors (at a $p$ value of 0.05 ). 
Table 1 Baseline Clinical Characteristics of Patients Not Taking Statins vs. Those Taking Statins Among Normoglycemic and Impaired Fasting Glucose (IFG) Patients

\begin{tabular}{|c|c|c|c|c|c|c|}
\hline & Normoglycemi & tients $(N=13,50$ & & IFG Patients & 4563) & \\
\hline & No statin & Statin & & No statin & Statin & \\
\hline Variable & $(N=9048)$ & $(N=4460)$ & $p$ value & $(N=2698)$ & $(N=1865)$ & $p$ value \\
\hline Age, years & $53.52 \pm 17.10$ & $60.12 \pm 13.60$ & $<0.001$ & $58.99 \pm 15.59$ & $62.18 \pm 12.39$ & $<0.001$ \\
\hline Age groups, $n(\%)$ & & & $<0.001$ & & & $<0.001$ \\
\hline $18-44$ & $2750(30 \%)$ & $573(13 \%)$ & & $463(17 \%)$ & $145(8 \%)$ & \\
\hline $45-64$ & $3943(44 \%)$ & $2173(49 \%)$ & & $1317(49 \%)$ & $924(50 \%)$ & \\
\hline $65+$ & $2355(26 \%)$ & $1714(38 \%)$ & & $918(34 \%)$ & $796(43 \%)$ & \\
\hline Male, $n(\%)$ & $3258(36 \%)$ & $2198(49 \%)$ & $<0.001$ & $1467(54 \%)$ & $1176(63 \%)$ & $<0.001$ \\
\hline Body mass index & $27.55 \pm 5.80$ & $28.31 \pm 4.91$ & $<0.001$ & $30.11 \pm 6.24$ & $30.08 \pm 5.29$ & 0.860 \\
\hline Systolic blood pressure, $\mathrm{mmHg}$ & $129.10 \pm 16.92$ & $130.40 \pm 16.25$ & $<0.001$ & $135.4 \pm 17.28$ & $134.40 \pm 16.07$ & 0.039 \\
\hline Diastolic blood pressure, $\mathrm{mmHg}$ & $77.33 \pm 9.65$ & $76.75 \pm 8.88$ & $<0.001$ & $79.98 \pm 10.04$ & $78.22 \pm 9.34$ & $<0.001$ \\
\hline Pulse, bpm & $75.08 \pm 10.50$ & $72.89 \pm 9.67$ & $<0.001$ & $75.72 \pm 10.87$ & $73.51 \pm 10.29$ & $<0.001$ \\
\hline Laboratory & & & & & & \\
\hline Total cholesterol, mg/dL & $198.8 \pm 32.13$ & $204.7 \pm 33.06$ & $<0.001$ & $201.1 \pm 31.63$ & $200.7 \pm 34.70$ & 0.700 \\
\hline HDL cholesterol, mg/dL & $57.43 \pm 15.95$ & $53.33 \pm 13.23$ & $<0.001$ & $51.91 \pm 14.85$ & $50.35 \pm 12.29$ & $<0.001$ \\
\hline LDL cholesterol, $\mathrm{mg} / \mathrm{dL}$ & $115.5 \pm 27.30$ & $119.7 \pm 31.14$ & $<0.001$ & $117.9 \pm 26.59$ & $115.1 \pm 29.05$ & 0.001 \\
\hline Triglycerides, $\mathrm{mg} / \mathrm{dL}$ & $126.1 \pm 64.24$ & $149.2 \pm 68.90$ & $<0.001$ & $153.4 \pm 78.16$ & $164.9 \pm 67.70$ & $<0.001$ \\
\hline Creatinine, $\mathrm{mg} / \mathrm{dL}$ & $1.01 \pm 0.26$ & $1.07 \pm 0.23$ & $<0.001$ & $1.05 \pm 0.22$ & $1.10 \pm 0.24$ & $<0.001$ \\
\hline Hemoglobin Alc, \% & $5.34 \pm 0.38$ & $5.48 \pm 0.52$ & $<0.001$ & $5.62 \pm 0.51$ & $5.67 \pm 0.43$ & 0.043 \\
\hline Fasting glucose, $\mathrm{mg} / \mathrm{dL}$ & $91.7 \pm 5.39$ & $93.14 \pm 4.67$ & $<0.001$ & $106.2 \pm 5.08$ & $106.2 \pm 4.91$ & 0.910 \\
\hline Hypertension medication use & & & & & & \\
\hline ACE inhibitors and ARB, $n(\%)$ & $1813(20 \%)$ & $1179(26 \%)$ & $<0.001$ & $754(28 \%)$ & $617(33 \%)$ & $<0.001$ \\
\hline Beta blockers, $n(\%)$ & $2531(28 \%)$ & $1461(33 \%)$ & $<0.001$ & $995(37 \%)$ & $794(43 \%)$ & $<0.001$ \\
\hline Calcium channel blocker, n (\%) & $1054(12 \%)$ & $604(14 \%)$ & 0.002 & $363(13 \%)$ & $303(16 \%)$ & 0.009 \\
\hline Diuretics, $n(\%)$ & $2001(22 \%)$ & $1012(23 \%)$ & 0.450 & $840(31 \%)$ & $542(29 \%)$ & 0.130 \\
\hline Other, $n(\%)$ & $274(3 \%)$ & $115(3 \%)$ & 0.140 & $107(4 \%)$ & $60(3 \%)$ & 0.190 \\
\hline Any of the above, $n(\%)$ & $4990(55 \%)$ & $2358(53 \%)$ & 0.012 & $1780(66 \%)$ & $1196(64 \%)$ & 0.200 \\
\hline Cholesterol medication use & & & & & & \\
\hline Fibrates, $n(\%)$ & $190(2 \%)$ & $209(5 \%)$ & $<0.001$ & $99(4 \%)$ & $130(7 \%)$ & $<0.001$ \\
\hline Statins, $n(\%)$ & $0(0 \%)$ & $4460(100 \%)$ & $<0.001$ & $0(0 \%)$ & $1865(100 \%)$ & $<0.001$ \\
\hline Other, $n(\%)$ & $209(2 \%)$ & $355(8 \%)$ & $<0.001$ & $64(2 \%)$ & $146(8 \%)$ & $<0.001$ \\
\hline Any of the above, $n(\%)$ & $388(4 \%)$ & $4460(100 \%)$ & $<0.001$ & $157(6 \%)$ & $1865(100 \%)$ & $<0.001$ \\
\hline Other medications & & & & & & \\
\hline Aspirin, $n(\%)$ & $5748(64 \%)$ & $2603(58 \%)$ & $<0.001$ & $1585(59 \%)$ & $1168(63 \%)$ & 0.008 \\
\hline Prior conditions & & & & & & \\
\hline Hypertension, $n(\%)$ & $3860(43 \%)$ & $2233(50 \%)$ & $<0.001$ & $1594(59 \%)$ & $1147(62 \%)$ & 0.100 \\
\hline Obesity, $n(\%)$ & $1830(20 \%)$ & $1007(23 \%)$ & 0.002 & $687(25 \%)$ & $494(26 \%)$ & 0.440 \\
\hline Tobacco, $n(\%)$ & $1266(14 \%)$ & $745(17 \%)$ & $<0.001$ & $476(18 \%)$ & $405(22 \%)$ & $<0.001$ \\
\hline Renal failure, $n(\%)$ & $273(3 \%)$ & $199(4 \%)$ & $<0.001$ & $116(4 \%)$ & $99(5 \%)$ & 0.110 \\
\hline Ischemic heart disease [HD], $n(\%)$ & $674(7 \%)$ & $1353(30 \%)$ & $<0.001$ & $255(9 \%)$ & $681(37 \%)$ & $<0.001$ \\
\hline Cerebrovascular disease, $n(\%)$ & $386(4 \%)$ & $313(7 \%)$ & $<0.001$ & $143(5 \%)$ & $159(9 \%)$ & $<0.001$ \\
\hline Hyperlipidemia, $n(\%)$ & $2562(28 \%)$ & $4153(93 \%)$ & $<0.001$ & $955(35 \%)$ & $1738(93 \%)$ & $<0.001$ \\
\hline Peripheral vascular disease, $n(\%)$ & $166(2 \%)$ & $235(5 \%)$ & $<0.001$ & $64(2 \%)$ & $93(5 \%)$ & $<0.001$ \\
\hline Congestive heart failure, $n(\%)$ & $218(2 \%)$ & $184(4 \%)$ & $<0.001$ & $85(3 \%)$ & $84(5 \%)$ & 0.017 \\
\hline Carotid disease, $n(\%)$ & $119(1 \%)$ & $175(4 \%)$ & $<0.001$ & $33(1 \%)$ & $92(5 \%)$ & $<0.001$ \\
\hline
\end{tabular}

Continuous variables are mean $\pm S D$. Categorical variables are number of patients and percentage

The Cox proportional hazards model with the total mortality endpoint was constructed using the above lipid risk propensity score, diagnoses of various cardiac and vascular complications, renal failure, hypertension and tobacco use. Backwards elimination was used to select a set of significant variables. The baseline lipid risk and the cholesterol drugs (statins and fibrates) were included regardless of whether they were significant. The Cox model with the diabetes endpoint was constructed using the lipid risk variable, various cardiac and vascular complications, renal disease, tobacco use, and fasting plasma glucose, and backwards elimination was similarly applied to arrive at a set of significant variables.

The survival curves were estimated using the Kaplan-Meier method and adjusting for baseline clinical characteristics (Table 1). The log-rank test was used to compare the survival curves, and $p$ values less than 0.05 were considered statistically significant. Statistical analyses were performed using SAS version 9.3 software (SAS institute Inc., Cary, NC, USA).

A sensitivity analysis was used to computationally validate the results from our models. A logistic propensity score model was constructed with statin use as outcome and the binarized risk factors as independent variables. The laboratory results were binarized as normal or abnormal using specific cutoffs. The study cohorts were then divided into subpopulations based on the binarized risk factors found to be significant in the propensity score model, in decreasing order of importance (decreasing order of their absolute coefficient). The result of this process was a non-overlapping partitioning of patients into groups with identical propensity scores and identical risk factors. In each group, the mean mortality and prevalence of diabetes was computed separately for the statin-taking and 
non-statin-taking patients. The difference between these means can be attributed to statin use. Groups that did not contain at least five mortality or diabetes events among either the statin-taking or non-statin-taking patients were discarded. The results obtained from this validation process were consistent with the results from the Cox models.

\section{RESULTS}

A total of 18,071 adult non-diabetic Olmsted County residents were taking some medication and had at least one outpatient fasting glucose value reported between 1999 and 2004. Of these, 13,508 were normoglycemic ( $n=4460 ; 33 \%$ taking statin) and 4563 met the criteria for IFG ( $n=1865 ; 41 \%$ taking statin).

These subjects were followed subsequently for a mean of 6 years. The baseline characteristics of these groups are summarized in Table 1. In general, IFG patients were older and had more cardiovascular risk factors, especially among those taking statins.

\section{Diabetes Risk}

After a mean 6 years of follow-up, there were $1182(10 \%)$ new diagnoses of diabetes in the normoglycemic group and $1524(36 \%)$ in the IFG group $(p<0.0001)$. Figure $1 \mathrm{a}$ and $\mathrm{b}$ shows the Kaplan-Meier curves and log-rank test $p$ value for the proportion of new diagnoses of diabetes after adjusting for baseline characteristics among normoglycemic and IFG patients. As expected, IFG patients had a higher risk of developing diabetes compared with normoglycemic patients. However, statin use was associated with increased risk of incident diabetes in both groups of patients, normoglycemic and IFG, although the difference between statin and non-statin users was more pronounced among the IFG patients (Fig. 1).

Table 2 shows the results of the Cox proportional hazards regression models. After adjustment for baseline characteristics, statin use was an independent predictor of incident diabetes in both patient groups, normoglycemic and IFG, with hazard ratios (HR) of 1.19 (95\% CI, 1.05 to 1.35$)$ and 1.24 (95\% CI, 1.11 to 1.38 ), respectively.

\section{Mortality Risk}

Over 6 years of follow-up, there were $926(7.2 \%)$ deaths in the normoglycemic group, compared with $450(10 \%)$ in the IFG group. Figure 1c and d shows Kaplan-Meier survival curves for overall survival after adjusting for baseline characteristics. Overall, patients with IFG had an increased risk of death compared with normoglycemic subjects. In both groups, however, patients taking statins had a statistically significant lower risk of death than patients not taking statins: HR $0.70(95 \%$ CI, 0.62 to 0.80 ) in the normoglycemic group vs. HR 0.77 (95\% CI, 0.64 to 0.91 ) in the IFG group (Table 3).

\section{DISCUSSION}

Our study findings indicated a positive association between statin use and an increased risk of incident diabetes in both normoglycemic and IFG patients, while overall mortality decreased with the use of statins in both of these populations. The risk of progression to overt diabetes in IFG patients over a 6-year period of follow-up was $24 \%$, a magnitude consistent with that reported in previous studies in similar populations, ${ }^{20-22}$ and as expected, the risk was higher for IFG patients than for normoglycemic subjects. However, this increased risk in incident diabetes was outweighed by an equal $24 \%$ reduction in overall mortality, a more important clinical outcome. Such excess risk of incident diabetes appears to be lower (19\%) in normoglycemic subjects with other cardiovascular risk factors, but the benefit of reduced mortality $(30 \%)$ in this group also greatly outweighs this risk.

Statin use was reported in a recent meta-analysis to be associated with an increased risk of developing diabetes, although this excess risk (9\%) appears to be small (odds ratio [OR] 1.09; $95 \%$ CI 1.02 to 1.17 ) and is most apparent in older subjects ( $\geq 60$ years). ${ }^{3}$ Furthermore, results of the individual randomized controlled trials (RCTs) included in the meta-analysis varied substantially, with only 2 of the 13 trials (JUPITER and PROSPER) ${ }^{10,23}$ showing a statistically significant increase in risk; the other 11 studies showed only non-significant trends towards lower ${ }^{1,7,24,25}$ or higher incidence $^{3}$ of diabetes. Several subsequent meta-analyses have confirmed this association, ${ }^{2,3,12}$ while another analysis failed to demonstrate a clear relationship. ${ }^{11}$ These differences are likely based on the specific clinical trials that were included in the various meta-analyses, but other factors may also play a role in these differences. For example, some studies have suggested that this is a class effect inherent to all statins, ${ }^{26}$ whereas others indicated that it may be associated only with more potent statins (atorvastatin, simvastatin, rosuvastatin) ${ }^{27-29}$; several studies have also suggested a dose-dependent effect. ${ }^{2,10,27,29}$ In any event, the underlying mechanism explaining this deleterious effect is unclear, although it has been postulated that statin therapy may interfere with normal glucose metabolism and exacerbate glucose intolerance. ${ }^{30,31}$

Statins are recommended for the management of hypercholesterolemia in the primary and secondary prevention of cardiovascular events in patients at risk. ${ }^{10,32-35}$ Given that diabetes has been considered a cardiovascular disease (CVD) risk equivalent, ${ }^{36}$ the use of statins is strongly recommended in patients with diabetes or those at high risk of developing diabetes, including those with metabolic syndrome or prediabetes, ${ }^{33}$ in whom CVD is highly prevalent and a common cause of death ${ }^{19,37,38}$; statins have been shown to prevent cardiovascular events and reduce mortality in this population. ${ }^{7}$ A recently published study evaluated the risk of incident diabetes in statin users who 
A

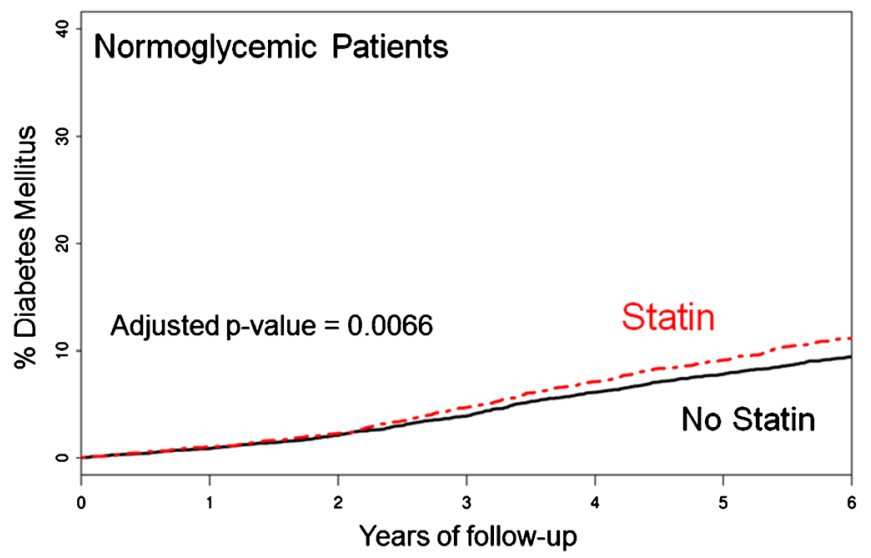

B

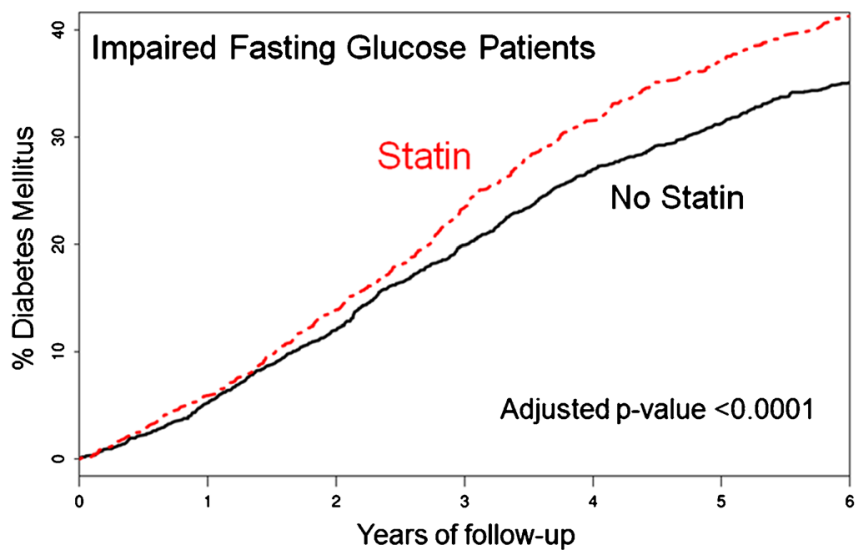

C

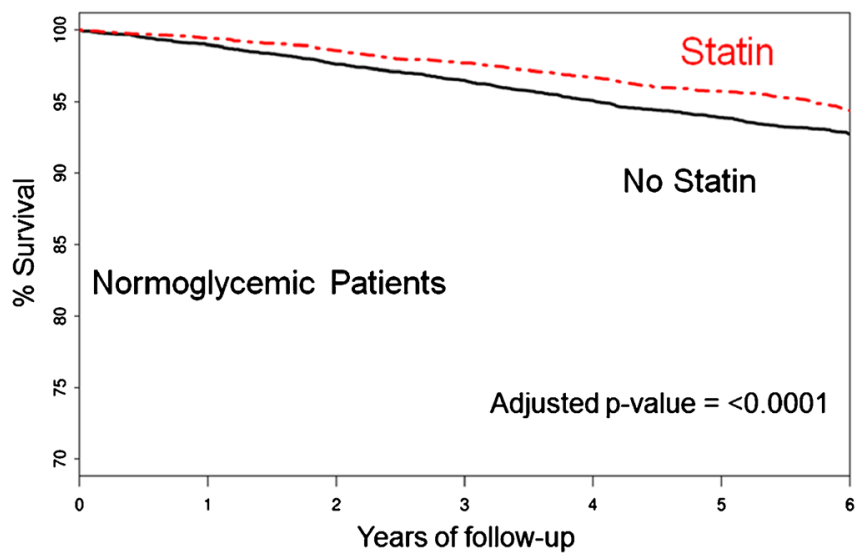

D

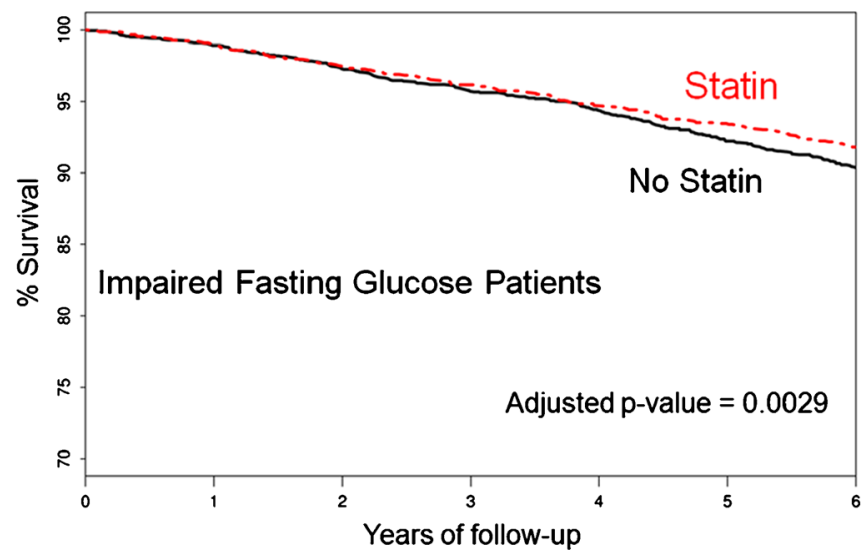

Figure 1 Kaplan-Meier curves and log-rank test $p$ value for the proportion of new diagnoses of diabetes (a and b) and overall survival (c and d) after adjusting for baseline characteristics among normoglycemic (a and c) and impaired fasting glucose patients (b and d).

had IGT and other cardiovascular risk factors at baseline, concluding that statin use in these patients was associated with a $32 \%$ increased risk of incident diabetes, ${ }^{13}$ although overall mortality in that group of statin users was not assessed. To the best of our knowledge, ours is the first study to directly evaluate the effects of statin use in a population of normoglycemic and IFG patients with regard to the risk of incident diabetes and, more importantly, overall mortality.
Prediabetes (IFG and IGT) is associated with increased risk of progression to overt diabetes, a well-known cardiovascular risk factor. ${ }^{22}$ Lifestyle and pharmacological interventions have been shown to reduce the rate of progression to diabetes in these high-risk populations. ${ }^{20,39}$ Because CVD is the leading cause of death in patients with diabetes, early intervention (at the prediabetes stage) may lead to a reduction in CVD. However, a recent meta-analysis, which included ten trials of interventions directed towards

Table 2 Predictors of Diabetes among Normoglycemic and Impaired Fasting Glucose (IFG) Patients

\begin{tabular}{|c|c|c|c|c|}
\hline \multirow[b]{2}{*}{ Predictor } & \multicolumn{2}{|l|}{ Normoglycemic Patients } & \multicolumn{2}{|l|}{ IFG Patients } \\
\hline & Hazard ratio \& $95 \%$ CI & $p$ value & Hazard ratio \& $95 \%$ CI & $p$ value \\
\hline Predictor & Hazard ratio \& $95 \%$ CI & $p$ value & Hazard ratio \& $95 \%$ CI & $p$ value \\
\hline Baseline lipid risk & $1.362(0.786,2.360)$ & 0.2704 & $1.471(1.008,2.148)$ & 0.0456 \\
\hline Male & $0.844(0.741,0.961)$ & 0.0107 & $0.841(0.751,0.943)$ & 0.0029 \\
\hline Age & $1.002(0.997,1.008)$ & 0.4509 & $0.994(0.989,0.998)$ & 0.0062 \\
\hline Body mass index & $1.047(1.037,1.058)$ & $<0.0001$ & $1.023(1.013,1.032)$ & $<0.0001$ \\
\hline Smoking & $1.265(1.081,1.481)$ & 0.0034 & - & - \\
\hline Renal Failure & $1.317(0.995,1.742)$ & 0.0541 & - & - \\
\hline Fasting glucose & $1.113(1.096,1.130)$ & $<0.0001$ & $1.094(1.084,1.105)$ & $<0.0001$ \\
\hline Any hypertension medication & $1.410(1.149,1.730)$ & 0.0010 & $1.131(1.004,1.274)$ & 0.0430 \\
\hline Fibrates & $1.439(1.097,1.888)$ & 0.0085 & $1.533(1.249,1.882)$ & $<0.0001$ \\
\hline Statins & $1.191(1.050,1.350)$ & 0.0066 & $1.238(1.109,1.383)$ & 0.0001 \\
\hline
\end{tabular}

Cells that are empty indicate variables that were not selected in the backward elimination of the multivariate Cox proportional hazards model 
Table 3 Predictors of Overall Mortality among Normoglycemic and Impaired Fasting Glucose (IFG) Patients

\begin{tabular}{|c|c|c|c|c|}
\hline \multirow[b]{2}{*}{ Predictor } & \multicolumn{2}{|l|}{ Normoglycemic Patients } & \multicolumn{2}{|l|}{ IFG Patients } \\
\hline & Hazard ratio \& $95 \%$ CI & $p$ value & Hazard ratio \& $95 \%$ CI & $p$ value \\
\hline Baseline lipid risk & $0.470(0.201,1.096)$ & 0.0805 & $0.706(0.376,1.324)$ & 0.2777 \\
\hline Male & $1.324(1.162,1.508)$ & $<0.0001$ & $1.385(1.159,1.655)$ & 0.0003 \\
\hline Age & $1.126(1.118,1.135)$ & $<0.0001$ & $1.128(1.117,1.139)$ & $<0.0001$ \\
\hline Body mass index & $1.009(0.996,1.023)$ & 0.1692 & $1.007(0.988,1.026)$ & 0.4861 \\
\hline Smoking & $2.129(1.802,2.516)$ & $<0.0001$ & $2.766(2.230,3.431)$ & $<0.0001$ \\
\hline Hypertension & $0.884(0.754,1.037)$ & 0.1308 & - & - \\
\hline Ischemic heart disease & $1.272(1.107,1.461)$ & 0.0007 & - & - \\
\hline Cerebrovascular disease & $1.674(1.438,1.949)$ & $<0.0001$ & $1.505(1.204,1.881)$ & 0.0003 \\
\hline Peripheral vascular disease & $1.467(1.200,1.793)$ & 0.0002 & - & - \\
\hline Congestive heart failure & $1.914(1.604,2.284)$ & $<0.0001$ & $1.933(1.479,2.527)$ & $<0.0001$ \\
\hline Carotid disease & $1.341(1.056,1.702)$ & 0.0160 & - & - \\
\hline Any hypertension medication & $1.238(0.981,1.562)$ & 0.0722 & - & - \\
\hline Fibrates & $0.768(0.511,1.155)$ & 0.2050 & $0.650(0.386,1.094)$ & 0.1047 \\
\hline Statins & $0.701(0.615,0.800)$ & $<0.0001$ & $0.766(0.643,0.913)$ & 0.0029 \\
\hline
\end{tabular}

Cells that are empty indicate variables that were not selected in the backward elimination of the multivariate Cox proportional hazards model

diabetes prevention in patients with IGT and IFG, failed to show that such interventions resulted in a reduction in allcause or CVD mortality. ${ }^{40}$ Conversely, our study demonstrates that despite the increased risk of incident diabetes associated with statin use -19 and $24 \%$ in normoglycemic and IFG patients, respectively - a significant reduction in overall mortality was also observed in statin users vs. nonusers in both normoglycemic and IFG patients, by 30 and $24 \%$, respectively. Our findings are consistent with those reported in the JUPITER trial, which showed a $27 \%$ increased risk of developing diabetes with statin use vs. nonuse, but a $54 \%$ reduction in the risk of myocardial infarction, a $48 \%$ reduction in the risk of stroke, and $20 \%$ lower risk of death from any cause. ${ }^{10}$

The main strength of our study is the very large sample size and long-term follow-up that includes mortality data. Our model also carefully selected comparable groups from our community-based population to avoid common biases, including the selection of a significantly different and healthier control group and imputation of data when that is not indicated based on a biased distribution of missing data. The main limitations include the retrospective nature of the study and the need for electronic data collection without individual chart review, which was not possible due to the large sample size and long follow-up. However, the Mayo comprehensive EMR and the resources available from the REP database provide reassurance regarding the validity of the data. It is important to highlight that our results apply only to subjects with comorbidities who are taking medications. In our efforts to define comparable groups, patients not taking medications, who were usually younger and healthier, were not included. Another limitation was our inability to ascertain the duration of exposure to individual statins due to the ambulatory use of these medications and our retrospective electronic data collection.

In conclusion, our data suggest that statin use is associated with a statistically significant increase in the risk of incident diabetes in both IFG and normoglycemic subjects, but also, and most importantly, with a significant reduction in overall mortality in both groups. Current recommendations for the use of statins in patients with clinical indications and as a means to decrease cardiovascular mortality and death seem valid, even after considering the risk of incident diabetes.

In our view, appropriate clinical follow-up of non-diabetic patients exposed to statins, including annual fasting glucose measurements, seems to be a logical compromise. Moreover, discontinuation of statin use in patients with rising glycemia who are at risk for cardiovascular events does not appear to be warranted, based on the low overall risk of diabetes - but more importantly, on the significant benefit of reduced mortality.

\section{Acknowledgments:}

Funders: This publication was supported by NIH/NCRR CTSA Grant No. UL1 RR024150. Its contents are solely the responsibility of the authors and do not necessarily represent the official views of the NIH. The authors appreciate support from the Mayo Clinic Department of Medicine and the WRAP award.

Corresponding Author: Pedro J. Caraballo, MD; Mayo Clinic, 200 First Street $\mathrm{S} W$, Rochester, MN 55905, USA (e-mail: caraballo.pedro@mayo.edu).

Compliance with Ethical Standards:

Conflict of Interest: The authors declare that they do not have a conflict of interest.

All authors contributed to the study design, review of the data, results and analysis, and writing of the manuscript. PJC, GS and SSC designed and supervised the electronic data collection. GS and SSC performed the statistical analysis.

\section{REFERENCES}

1. Freeman DJ, Norrie J, Sattar N, et al. Pravastatin and the development of diabetes mellitus: evidence for a protective treatment effect in the West of Scotland Coronary Prevention Study. Circulation. 2001;103(3):357-62.

2. Preiss D, Seshasai SR, Welsh $\mathbf{P}$, et al. Risk of incident diabetes with intensive-dose compared with moderate-dose statin therapy: a metaanalysis. JAMA. 2011;305(24):2556-64.

3. Sattar N, Preiss D, Murray HM, et al. Statins and risk of incident diabetes: a collaborative meta-analysis of randomised statin trials. Lancet. 2010;375(9716):735-42. 
4. Cholesterol Treatment Trialists' Collaborators. The effects of lowering LDL cholesterol with statin therapy in people at low risk of vascular disease: meta-analysis of individual data from 27 randomised trials. Lancet. 2012;380(9841):581-90.

5. HPSC Group. Cost-effectiveness of simvastatin in people at different levels of vascular disease risk: economic analysis of a randomised trial in 20536 individuals. Lancet. 2012;365(9473):1779-85.

6. Collins R, Armitage J, Parish S, Sleigh P, Peto R. MRC/BHF Heart Protection Study of cholesterol-lowering with simvastatin in 5963 people with diabetes: a randomised placebo-controlled trial. Lancet. 2003;361(9374):2005-16.

7. Keech A, Colquhoun D, Best J, et al. Secondary prevention of cardiovascular events with long-term pravastatin in patients with diabetes or impaired fasting glucose: results from the LIPID trial. Diabetes Care. 2003;26(10):2713-21.

8. Sever PS, Dahlof B, Poulter NR, et al. Prevention of coronary and stroke events with atorvastatin in hypertensive patients who have average or lower-than-average cholesterol concentrations, in the Anglo-Scandinavian Cardiac Outcomes Trial-Lipid Lowering Arm (ASCOT-LLA): a multicentre randomised controlled trial. Lancet. 2003;361(9364):1149-58.

9. Kjekshus J, Apetrei E, Barrios V, et al. Rosuvastatin in older patients with systolic heart failure. N Engl J Med. 2007;357(22):2248-61.

10. Ridker PM, Danielson E, Fonseca FAH, et al. Rosuvastatin to prevent vascular events in men and women with elevated C-reactive protein. $\mathrm{N}$ Engl J Med. 2008;359(21):2195-207.

11. Rajpathak SN, Kumbhani DJ, Crandall J, Barzilai N, Alderman M, Ridker PM. Statin therapy and risk of developing type 2 diabetes: a metaanalysis. Diabetes Care. 2009;32(10):1924-29.

12. Waters DD, Ho JE, DeMicco DA, et al. Predictors of new-onset diabetes in patients treated with atorvastatin: results from 3 large randomized clinical trials. J Am Coll Cardiol. 2011;57(14):1535-45.

13. Shen L, Shah BR, Reyes EM, et al. Role of diuretics, beta blockers, and statins in increasing the risk of diabetes in patients with impaired glucose tolerance: reanalysis of data from the NAVIGATOR study. BMJ. 2013;347:f6745.

14. Abramson J, Wright JM. Are lipid-lowering guidelines evidence-based? Lancet. 2007;369(9557):168-69.

15. Brugts JJ, Yetgin T, Hoeks SE, et al. The benefits of statins in people without established cardiovascular disease but with cardiovascular risk factors: meta-analysis of randomised controlled trials. BMJ. 2009;338: b2376.

16. Mills EJ, Rachlis B, Wu P, Devereaux PJ, Arora P, Perri D. Primary prevention of cardiovascular mortality and events with statin treatments: a network meta-analysis involving more than 65,000 patients. J Am Coll Cardiol. 2008;52(22): 1769-81.

17. Ray KK, Seshasai SR, Erqou S, et al. Statins and all-cause mortality in highrisk primary prevention: a meta-analysis of 11 randomized controlled trials involving 65,229 participants. Arch Intern Med. 2010;170(12):1024-31.

18. Thavendiranathan P, Bagai A, Brookhart MA, Choudhry NK. Primary prevention of cardiovascular diseases with statin therapy: a meta-analysis of randomized controlled trials. Arch Intern Med. 2006;166(21):2307-13.

19. Goldfine AB. Statins: is it really time to reassess benefits and risks? N Engl J Med. 2012;366(19):1752-55.

20. Knowler WC, Barrett-Connor E, Fowler SE, et al. Reduction in the incidence of type 2 diabetes with lifestyle intervention or metformin. $\mathrm{N}$ Engl J Med. 2002;346(6):393-403.

21. Lindstrom J, Louheranta A, Mannelin M, et al. The Finnish Diabetes Prevention Study (DPS): lifestyle intervention and 3-year results on diet and physical activity. Diabetes Care. 2003;26(12):3230-36.

22. Tabak AG, Herder C, Rathmann W, Brunner EJ, Kivimaki M. Prediabetes: a high-risk state for diabetes development. Lancet. 2012;379(9833): 2279-90.
23. Shepherd J, Blauw GJ, Murphy MB, et al. Pravastatin in elderly individuals at risk of vascular disease (PROSPER): a randomised controlled trial. Lancet. 2002;360(9346):1623-30.

24. Downs JR, Clearfield M, Weis $\mathbf{S}$, et al. Primary prevention of acute coronary events with lovastatin in men and women with average cholesterol levels: results of AFCAPS/TexCAPS. Air Force/Texas Coronary Atherosclerosis Prevention Study. JAMA. 1998;279(20):1615-22.

25. Investigators G. Prevenzione. Results of the low-dose (20 mg) pravastatin GISSI Prevenzione trial in 4271 patients with recent myocardial infarction: do stopped trials contribute to overall knowledge? GISSI Prevenzione Investigators (Gruppo Italiano per lo Studio della Sopravvivenza nell'Infarto Miocardico). Ital Heart J. 2000;1(12):810-20.

26. Culver AL, Ockene IS, Balasubramanian R, et al. Statin use and risk of diabetes mellitus in postmenopausal women in the Women's Health Initiative. Arch Intern Med. 2012;172(2): 144-52.

27. Baker WL, Talati R, White CM, Coleman CI. Differing effect of statins on insulin sensitivity in non-diabetics: a systematic review and meta-analysis. Diabetes Res Clin Pract. 2010;87(1):98-107.

28. Carter AA, Gomes T, Camacho X, Juurlink DN, Shah BR, Mamdani MM. Risk of incident diabetes among patients treated with statins: population based study. BMJ. 2013;346:f2610.

29. Navarese EP, Buffon A, Andreotti F, et al. Meta-analysis of impact of different types and doses of statins on new-onset diabetes mellitus. Am J Cardiol. 2013;111(8):1123-30.

30. Kanda M, Satoh K, Ichihara K. Effects of atorvastatin and pravastatin on glucose tolerance in diabetic rats mildly induced by streptozotocin. Biol Pharm Bull. 2003;26(12):1681-84.

31. Sasaki J, Iwashita M, Kono S. Statins: beneficial or adverse for glucose metabolism. J Atheroscler Thromb. 2006;13(3): 123-29.

32. Kostis WJ, Cheng JQ, Dobrzynski JM, Cabrera J, Kostis JB. Metaanalysis of statin effects in women versus men. J Am Coll Cardiol. 2012;59(6):572-82.

33. Kearney PM, Blackwell L, Collins R, et al. Efficacy of cholesterol-lowering therapy in 18,686 people with diabetes in 14 randomised trials of statins: a meta-analysis. Lancet. 2008;371(9607):117-25.

34. Armitage J, Bowman L, Wallendszus $\mathbf{K}$, et al. Intensive lowering of LDL cholesterol with $80 \mathrm{mg}$ versus $20 \mathrm{mg}$ simvastatin daily in 12,064 survivors of myocardial infarction: a double-blind randomised trial. Lancet. 2010;376(9753): 1658-69.

35. Baigent C, Blackwell L, Emberson J, et al. Efficacy and safety of more intensive lowering of LDL cholesterol: a meta-analysis of data from 170,000 participants in 26 randomised trials. Lancet. 2010;376(9753): $1670-81$.

36. Haffner SM, Lehto S, Ronnemaa T, Pyorala K, Laakso M. Mortality from coronary heart disease in subjects with type 2 diabetes and in nondiabetic subjects with and without prior myocardial infarction. N Engl J Med. 1998;339(4):229-34.

37. Sarwar N, Gao P, Seshasai SR, et al. Diabetes mellitus, fasting blood glucose concentration, and risk of vascular disease: a collaborative metaanalysis of 102 prospective studies. Lancet. 2010;375(9733):2215-22.

38. Seshasai SR, Kaptoge S, Thompson A, et al. Diabetes mellitus, fasting glucose, and risk of cause-specific death. N Engl J Med. 2011;364(9): 829-41.

39. Gillies CL, Abrams KR, Lambert PC, et al. Pharmacological and lifestyle interventions to prevent or delay type 2 diabetes in people with impaired glucose tolerance: systematic review and meta-analysis. BMJ. 2007;334(7588):299.

40. Hopper I, Billah B, Skiba M, Krum H. Prevention of diabetes and reduction in major cardiovascular events in studies of subjects with prediabetes: meta-analysis of randomised controlled clinical trials. Eur $\mathrm{J}$ Cardiovasc Prev Rehabil. 2011;18(6):813-23. 